\title{
A double blind, placebo controlled, randomised pilot trial examining the effects of probiotic administration on mood and cognitive function
}

\author{
L. Owen ${ }^{1}$, M. Reinders ${ }^{2}$, R. Narramore ${ }^{3}$, A. M. R Marsh ${ }^{3}$, F. Gar Lui ${ }^{3}$, R. Baron ${ }^{3}$, \\ Sue Plummer ${ }^{4}$ and B. M. Corfe ${ }^{3}$ \\ ${ }^{1}$ School of Psychology, Keele University, UK, ${ }^{2}$ Utrecht University, Netherlands, ${ }^{3}$ Molecular Gastroenterology Research \\ Group, Academic Unit of Surgical Oncology, Department of Oncology, University of Sheffield, Royal Hallamshire \\ Hospital, Sheffield, UK and ${ }^{4}$ Cultech Limited, Christchurch Rd Port Talbot, Neath Port Talbot
}

There is a growing body of evidence indicating that the gut microbiota communicates with the CNS influencing mood and behaviour $^{(1-7)}$ and a role for the microbiota in the development of brain plasticity and the subsequent physiological response has been suggested ${ }^{(2)}$. Furthermore treatment with probiotics has been shown to alter functional task-related brain activity and changes in midbrain connectivity ${ }^{9}$. To date no study has demonstrated cognitive modification in response to probiotic treatment. The aims of the study were i) To examine the mood effects of probiotic supplementation at rest and in response to psychological stressors ii) To examine the effects of probiotic administration on cognitive functioning.

In this pilot study, healthy participants $(n=50)$ were recruited to take part in a double blind, randomised, controlled trial. Participants were randomized to receive either a probiotic preparation comprising two strains of Lactobacillus acidophilus CUL60 (NCIMB 30157) and CUL21 (NCIMB 30156), Bifidobacterium lactis CUL34 (NCIMB 30172) and Bifidobacterium bifidum CUL20 (NCIMB 30153) at a total of $2.5 \times 10^{10} \mathrm{cfu} /$ capsule or a placebo for 6 weeks. The sample population comprised 18 males and 32 females, mean age 23.22 years (range 19-38, sD 3.846), with a BMI <30. Participants underwent morphometric measurements (height, weight, \% total body fat, hip and waist measurement) and completed mood, stress and depression questionnaires (Bond Lader Mood Scales, Stait Trait Anxiety Inventory and NASA Task Load Index). Participants also were required to perform a battery of computerised cognitive test (COMPASS) measuring attention, executive function, working memory and episodic memory.

In terms of mood measures, a significant interaction showed that 'trait' anxiety levels decreased in the active probiotic condition whilst increasing in the placebo condition over the course of the 6 week intervention $(p=0.042)$. A significant interaction was also observed for tasks of attention; 'continuity of attention increased in response to the probiotic treatment and decreased with the placebo group $(p=0.035)$. No morphometric changes between the two treatment conditions were recorded during the intervention period. The findings of this pilot trial provide a justification for further studies to characterise the potential cognitive and mood enhancing benefits of probiotics in healthy populations.

This work was supported by Bioceticals, NSW Australia and Cultech Limited, S. Wales, UK.

1. Cryan JF \& Dinan TG (2012) Nat. Rev. Neurosci. 13, 701-712.

2. Sudo $\mathrm{N}$ et al. (2004) The Journal of physiology 558, 263-275.

3. O'Mahony L et al. (2005) Gastroenterology. 128, 541-551.

4. Gaykema R et al. (2004) Brain. Behav. Immun. 18, 238-245.

5. Goehler LE et al. (2005) Brain. Behav. Immun. 19, 334-344.

6. Goehler LE et al. (2008) Brain. Behav. Immun. 22, 354-366.

7. Lyte M et al. (2006) Physiol. Behav. 89, 350-357. 